\title{
Sustentabilidade agrícola e saúde do solo
}

O solo constitui-se em um recurso natural complexo de extrema importância para a produção agrícola, seu manejo sustentável reflete em seu potencia produtivo. Deste modo, objetivou-se identificar práticas relacionadas a sustentabilidade agrícola e a manutenção da saúde do solo, com a finalidade de gerar informações que auxiliem pesquisas futuras, bem como para nortear produtores quanto as práticas a serem implementadas visando a conservação ambiental. De acordo com o levantado a agricultura conservacionista apresenta-se como uma forte opção para o alcance da sustentabilidade agrícola, pois a degradação do solo está relacionada com modificações em seus atributos físicos, químicos e biológicos, que podem ocorrer de forma natural, ou pela ação do homem, derivada de práticas inadequadas de manejo. Dentre as ações que podem promover a saúde do solo destacam-se a adoção de plantio direto ou mínimo revolvimento do solo; a cobertura permanente do solo pelo cultivo de plantas para sua proteção e adição de nutrientes, combinados com rotação e/ ou consórcio de culturas que atuam na reciclagem de nutrientes por meio da decomposição de resíduos das plantas cultivadas, ou das plantas de cobertura; e a implantação de terraços, prática que contribui para a contenção e atraso das águas que carreiam nutrientes e agroquímicos aos mananciais hídricos. A divulgação destas técnicas amplia as alternativas na busca pelo desenvolvimento de uma agricultura sustentável.

Palavras-chave: Manejo e conservação; Meio ambiente; Práticas conservacionistas.

\section{Agricultural sustainability and soil health}

The soil is a complex natural resource of extreme importance for agricultural production, its sustainable management reflects in its productive potential. Thus, the objective was to identify practices related to agricultural sustainability and the maintenance of soil health, with the purpose of generating information to assist future research, as well as to guide producers as to the practices to be implemented aiming at environmental conservation. According to the survey, conservation agriculture presents itself as a strong option for achieving agricultural sustainability, since soil degradation is related to changes in its physical, chemical and biological attributes, which can occur naturally, or by action of man, derived from inadequate management practices. Among the actions that can promote soil health, the adoption of direct planting or minimal soil tillage stands out; the permanent cover of the soil by the cultivation of plants for their protection and addition of nutrients, combined with rotation and / or consortium of cultures that act in the recycling of nutrients through the decomposition of residues of the cultivated plants, or of the cover plants; and the implantation of terraces, a practice that contributes to the containment and delay of waters that carry nutrients and agrochemicals to water sources. The dissemination of these techniques expands the alternatives in the search for the development of sustainable agriculture.

Keywords: Management and conservation; Environment; Conservationist practices.

Topic: Ciências do Solo

Reviewed anonymously in the process of blind peer.

Luiz Henrique dos Reis Bocaleti (iD

Universidade Cesumar, Brasil

http://lattes.cnpq.br/9185284432984159

http://orcid.org/0000-0003-0034-9280

bocaletiluizh@hotmail.com

Francielli Gasparotto (iD

Universidade Cesumar, Brasil

http://lattes.cnpq.br/2673470812353146

https://orcid.org/0000-0002-4038-7364

francipg@gmail.com

Sabrina Pariz

Universidade Cesumar, Brasil

http://lattes.cnpq.br/1773983062439048

sa pariz@hotmail.com
Received: 04/04/2021

Approved: 01/05/2021

Edison Schmidt Filho

Universidade Cesumar, Brasil

http://lattes.cnpq.br/7605628504842582

edison.schmidt@unicesumar.edu.br

Edneia Aparecida de Souza Paccola (it)

Universidade Cesumar, Brasil

http://lattes.cnpq.br/5090759600495959

http://orcid.org/0000-0002-3182-3224

edneia.paccola@unicesumar.edu.br

\section{Referencing this:}

BOCALETI, L. H. R.; GASPAROTTO, F.; PARIZ, S.; SCHMIDT FILHO, E.; PACCOLA, E. A. S.. Sustentabilidade agrícola e saúde do solo. Revista Ibero Americana de Ciências Ambientais, v.12, n.5, p.1-13, 2021. DOI: http://doi.org/10.6008/CBPC2179-6858.2021.005.0001 


\section{INTRODUÇÃO}

O solo é um recurso natural não renovável composto por partículas minerais tais como: areia, silte e argila, além de matéria orgânica, água, ar e organismos vivos. É o suporte para toda a produção agrícola, sendo o seu equilíbrio um fator primordial para o potencial produtivo e para a sustentabilidade agrícola (SCHOONOVER et al., 2015).

Estima-se que, até 2100 , a população mundial ultrapasse a marca de 10,0 bilhões de habitantes, refletindo em maior demanda global por alimentos e consequente uso exacerbado do solo (ONU, 2017). Contudo, a produção para atender esta demanda deve ser pautada em sistemas de produção agrícola sustentáveis visando promover baixos impactos ambientais (SARKAR et al., 2017; RAKSHIT, 2019). Neste sentido, um desafio é o aumento e o uso intensificado dos serviços ecossistêmicos fornecidos diretamente pelo solo, assim, mudanças são necessárias no que se refere ao uso e cobertura do solo, buscando reduzir sua degradação e diminuir o fluxo de $\mathrm{CO}_{2}$ (DUBEY et al., 2020).

Sarkar et al. (2020) apontam que um terço do solo da Terra apresenta condições severas de degradação relacionada a vários processos, por exemplo: a intensificação agrícola, a poluição do solo, a erosão do solo, e a má gestão de recursos e desertificação. Telles et al. (2019) relatam que 40\% do solo usado para a produção agrícola mundial está severamente degradado devido a gestão inadequada. Supõese que 12 milhões de hectares correspondentes a terras agrícolas são perdidos devido ao aumento na degradação do solo, resultando na reduzida capacidade de produção. Dessa forma, a crescente demanda mundial por alimento exige que terras poluídas/ou degradadas sejam utilizadas para a produção agrícola através de práticas ecológicas adequadas (ABHILASH et al.; 2016; SARKAR et al.; 2017).

Destaca-se que os recursos naturais precisam ser geridos de forma sustentável e racional. Tais medidas adaptativas devem ser economicamente, ecologicamente e socialmente aceitáveis com o objetivo de obter sistemas agrícolas de produção sustentáveis e com redução de impactos ambientais (SARKAR et al., 2020). Neste sentido, a adoção de tecnologias sustentáveis no manejo do solo contribui com sua preservação e de acordo com Busari et al. (2015) podem proporcionar a manutenção da estrutura e da conservação de água no solo, a redução dos riscos de erosão e das flutuações na temperatura, além de melhorar a qualidade do solo.

Porém, apesar do amplo número de técnicas de manejo do solo, os produtores rurais nem sempre reconhecem ou conseguem identificar a melhor opção a ser adotada em sua propriedade. Charel et al. (2018) observaram que a percepção e o conhecimento dos produtores com relação a saúde do solo em uma região da Índia evitaram a adição excessiva de fertilizantes, e consequente degradação contínua dos recursos naturais sob agricultura intensiva. Diante do exposto, o presente trabalho de revisão visou identificar práticas relacionadas a sustentabilidade agrícola e a manutenção da saúde do solo, com a finalidade de gerar informações que auxiliem pesquisas futuras, bem como para nortear produtores quanto as práticas a serem implementadas visando a conservação ambiental. 


\section{METODOLOGIA}

O estudo trata-se de uma revisão narrativa ou tradicional, pois apresenta uma temática mais aberta. O presente trabalho foi realizado por meio de um levantamento bibliográfico mediante consulta às bases de dados online, principalmente de revistas indexadas, tais como: Scielo, Web Of Science, além de teses e dissertações. Foram selecionados artigos escritos em inglês, português ou espanhol.

$\mathrm{Na}$ busca de artigos, foram utilizados, os seguintes descritores e suas combinações na língua portuguesa e inglesa respectivamente: solos; manejo de solos; manejo e conservação; práticas conservacionistas; saúde do solo; sustentabilidade agrícola. Os critérios de inclusão para a seleção de artigos foram às combinações de palavras acima, relacionadas com a sustentabilidade agrícola, saúde do solo e a mitigação dos impactos das práticas agrícolas.

\section{REVISÃO TEÓRICA}

\section{Agricultura e sustentabilidade}

A agricultura é uma atividade milenar, instituída a partir do momento que o homem abandonou as práticas nômades de caça e coleta, e passou a produzir alimento para a sua subsistência, por meio da criação de animais, seleção de vegetais e cultivo no solo (THRALL et al., 2010). Assim, a agricultura se tornou uma prática intencional, e o solo começou a ser utilizado para o cultivo de espécies previamente selecionadas, o que concomitantemente, gerou alterações em seus atributos físicos, químicos e biológicos (SINGH et al., 2017).

Todavia, dos primórdios à atualidade, os sistemas agrícolas passaram por mudanças conceituais e tecnológicas decorrentes de transformações econômicas, sociais e ambientais (YAN et al., 2020), contribuindo com a alta produtividade por meio de práticas como a monocultura, mecanização agrícola para o cultivo intensivo do solo, e utilização de agroquímicos, tais como: fertilizantes sintéticos, inseticidas e herbicidas (ANGELAKIS et al., 2020). Infelizmente, algumas práticas empregadas, embasadas na economia de mercado e inovação tecnológica, resultaram na degradação de ecossistemas naturais, erosão, e redução da fertilidade dos solos (HARIZANOVA et al., 2019).

Devido aos impactos socioambientais, surgiram questionamentos relacionados a práticas agrícolas mais sustentáveis, direcionadas à segurança na produção de alimentos, e a preservação ambiental (SANTOS et al., 2019). Os autores ainda explicam que a agricultura sustentável busca rendimentos em longo prazo, por meio do uso de tecnologias de manejo ecologicamente mais adequadas. Nesse contexto, o desenvolvimento da sustentabilidade está associado com três categorias: sustentabilidade do sistema econômico, sustentabilidade do sistema ambiental, e sustentabilidade do sistema social (DURAN et al.; 2015).

A sustentabilidade econômica possibilita a alocação e gestão eficiente dos recursos produtivos e o fluxo regular de investimentos públicos e privados. Esta, não se limita ao capital monetário, mas passa e integra-se por diferentes bases, como capital físico, financeiro, humano, intelectual, ambiental e/ou natural e social. Nesse contexto, a sustentabilidade econômica na agricultura configura-se como a capacidade de 
gerar produtos de valor comercial com valor agregado, por meio da adoção de sistemas de produção e cultivo que minimizem perdas e desperdícios, com produtividade compatível com os investimentos realizados, com mecanismos que possibilitem a competitividade no mercado agrícola garantindo a economicidade na cadeia produtiva, a qualidade do produto, e a satisfação e manutenção na atividade desenvolvida (BARBIERI et al., 2016).

Já a sustentabilidade ambiental é caracterizada pela ampliação da capacidade de gerar recursos naturais renováveis, restringindo o uso dos recursos naturais não renováveis, reduzindo as emissões de gases poluentes, e ampliando a pesquisa e o uso de tecnologias limpas para a proteção ambiental (BARBIERI et al., 2016). Por fim, os autores ainda afirmam que a sustentabilidade social na agricultura é a consolidação de processos capazes de promover a equidade na distribuição dos bens e renda para melhorar os direitos e condições de vida das populações, reduzindo as desigualdades sociais.

Entre os fatores que influenciam e direcionam a sustentabilidade social na agricultura destacam-se os costumes, tradições, espiritualidade, cultura, relações interpessoais e condições de vida na sociedade incluindo: pobreza, violência, educação, trabalho, saúde pública e direitos humanos. Assim, a sustentabilidade social na agricultura refere-se a um processo de desenvolvimento que leva a um crescimento estável com distribuição equitativa de renda, diminuindo as diferenças entre os diversos níveis na sociedade e melhorando a condição de vida da população (CZYZEWSKI et al., 2018; JANKER et al., 2019).

A avaliação da sustentabilidade na agricultura não se fundamenta apenas nas questões ambientais e técnicas, mas integra a multifuncionalidade das questões econômicas e sociais. Um dos desafios da sustentabilidade na agricultura envolve a compreensão das necessidades atuais sem o comprometimento das gerações futuras (BIDONE et al., 2004).

Dentre outros propósitos para a agricultura sustentável destacam-se: dobrar a produtividade agrícola e a renda dos pequenos produtores de alimentos por meio do acesso seguro e igual à terra, outros recursos produtivos e insumos, conhecimento, serviços financeiros, mercados e oportunidades de agregação de valor e de emprego não agrícola. Implementar práticas agrícolas resilientes que ajudem a manter os ecossistemas, que fortaleçam a capacidade de adaptação as mudanças climáticas, às condições meteorológicas extremas, secas, inundações, e que melhorem progressivamente a qualidade da terra e do solo (ONU, 2015).

Um ecossistema saudável protege o planeta, e permite a subsistência humana. Florestas, montanhas e pântanos, por exemplo, são responsáveis pelo ar limpo, pela água, pela conservação da biodiversidade e mitigação da mudança climática. As florestas e pastagens provém matéria prima para as indústrias, gera empregos e rendimentos, colabora com a produção de alimento, com a medicina e com a produção de combustível para mais de 1 bilhão de pessoas (FAO et al., 2020).

Ainda segundo a Organização os recursos naturais estão se deteriorando, os ecossistemas estão em desequilíbrio, e a diversidade biológica está sendo perdida no planeta. Mudanças no uso da terra, incluindo o desflorestamento é responsável pela perda de valiosos habitats, redução da água potável, degradação da terra, erosão do solo e liberação de carbono para a atmosfera. Para que a sustentabilidade agrícola seja 
alcançada é necessário que o homem mude sua relação com o meio ambiente e principalmente implemente práticas de manejo de solo adequadas.

\section{Qualidade do solo}

A palavra solo apresenta muitos significados, de acordo com Fao et al. (2020) o significado tradicional de solo é o meio natural para o crescimento das plantas. Van Es (2017) propôs uma definição de solo inclusiva, direta, precisa, sucinta, e que usa uma linguagem comum para todos. Segundo o autor supracitado solo é:

A(s) camada (s) de mineral geralmente solto e/ou material orgânico que são afetados por processos físicos, químicos e/ou biológicos na ou perto da superfície planetária, geralmente contêm líquidos, gases, biota e plantas suporte.

O solo é um recurso natural complexo e heterogêneo, essencial à manutenção do meio ambiente e ecossistemas, é formado a partir de um material semi consolidado oriundo do processo de intemperismo físico, químico e biológico sofrido pelas rochas. O solo desempenha funções ecológicas, como por exemplo o acúmulo da energia solar na forma de matéria orgânica, reciclagem da água e nutrientes, suporte para o crescimento de inúmeras espécies da fauna e flora, regulador dos ciclos de elementos químicos, como carbono, nitrogênio, potássio, enxofre e fósforo, e matéria-prima para artefatos humanos (PARIKH et al., 2012; AHLAWAT et al., 2020).

Quando os atributos físicos, químicos e biológicos do solo são modificados, diz-se que ocorreu a degradação do solo, com perda da capacidade de produção dentro de um ecossistema, podendo ocorrer de forma natural, ou a partir da interferência antrópica por meio de práticas inadequadas de manejo. Assim, a saúde do solo não é afetada apenas por sua gênese ou formação, mas também por fatores relacionados ao uso e manejo, que contribuem com sua degradação, como as práticas agrícolas inadequadas, incluindo cultivos e pastoreio intensivos, contaminação com agroquímicos e o desmatamento (GHOLOUBI et al., 2019; DEY et al., 2019).

A conservação adequada no uso do solo é de responsabilidade de todos, dos proprietários, dos responsáveis por assistência técnica, das instituições de pesquisa, dos órgãos ambientais, das instituições financeiras, e dos administradores públicos de áreas urbanas ou rurais (VERMA et al., 2019). Mesquita; Cruz; Pinheiro (2016) afirmam que o manejo e a conservação dos recursos naturais, tais como o solo, tem maior equilíbrio quando associado as florestas nativas, pois, protege os sistemas bióticos e/ou abióticos, restaura as propriedades químicas e físicas, aumenta a matéria orgânica, e a qualidade do solo.

As lavouras afetam as propriedades físicas, químicas e biológicas do solo em diferentes magnitudes, refletindo na forma de escoamento superficial e ocorrência de erosões do solo (BHATT et al., 2006). O preparo do solo, ou seja, sua manipulação mecânica para a produção agrícola, afeta significativamente suas características, como temperatura, processos relacionados a conservação da água, infiltração e evapotranspiração (BUSARI et al., 2015). Porém, o uso do solo é inevitável, pois é nele que os alimentos são cultivados, ele também atua indiretamente em outros diversos processos, como a extração e formulação de substâncias e medicamentos, fibra têxtil, materiais para construção civil, além de ter relação 
com os ciclos do meio ambiente, principalmente o ciclo da água (COSTA et al., 2020).

Nesse sentido, a agricultura conservacionista surge como um componente chave para os desafios globais decorrentes do aumento da produção de alimento através da conservação do meio ambiente (DEY et al., 2019). Além disso, alguns estudos consideram que a agricultura conservacionista é chave para preconizar a adoção de plantio direto ou mínimo revolvimento do solo, a cobertura permanente do solo pelo cultivo de plantas para sua proteção e adição de nutrientes, combinados com rotação e/ ou consórcio de culturas que atuam na reciclagem de nutrientes por meio da decomposição de resíduos das plantas cultivadas, ou das plantas de cobertura (HOBB et al., 2008).

De forma geral, as técnicas de conservação do solo podem ser divididas em duas categorias: a conservação biológica e, a conservação mecânica. Em suma, a primeira visa aumentar a saúde do solo, melhorando o conteúdo de matéria orgânica, as propriedades físicas e o status nutricional do solo, além de ser uma técnica rápida, barata, não exigindo o uso de terraços ou outras estruturas permanentes (SINORE et al., 2018). Exemplos de técnicas de conservação biológica do solo são: aplicação de esterco e adubos verdes, uso de espécies de gramíneas ( $P$. purpureum e $S$. sesban), rotação de culturas, e uso de barreiras vegetais (SARMINAH et al., 2018; SINORE et al., 2018).

Ora as técnicas de conservação mecânica têm por objetivo proteger o solo dos impactos causados pela chuva pesada e vento, e prevenir contra a erosão. Segundo Fao et al. (2020), estas técnicas baseiam-se na modificação da inclinação do solo, influencia o escoamento superficial e permite o uso agrícola de encostas íngremes. Exemplos desta técnica incluem: uso de terraços, canais de drenagem, e barreiras de pedras/rochas.

Ressalta-se que a qualidade do solo é um dos três componentes da qualidade ambiental, e diferente da qualidade da água e do ar, não está limitada ao grau de poluição, mas é comumente definida como a capacidade do solo funcionar dentro do ecossistema e dos limites de uso da terra sustentando a produtividade biológica, mantendo a qualidade ambiental, e promovendo a saúde das plantas e animais. A qualidade do solo é mais complexa que a qualidade do ar e da água, não só por conta dos constituintes do solo, mas porque o solo pode ser usado para uma variedade de propósitos. Os solos geralmente reagem lentamente as mudanças no uso da terra e manejo, dessa forma, torna-se mais difícil detectar mudanças na qualidade do solo antes de danos mais severos (BÜNEMANN et al., 2018).

Doran et al. (1994) foram os pioneiros na busca do melhor índice de qualidade do solo. Existem um conjunto de indicadores de ordem química, física e biológica para avaliar a qualidade do solo, os quais incluem: textura, profundidade do solo e das raízes, densidade do solo, infiltração de água no solo, capacidade de armazenamento, retenção de água, conteúdo de água no solo, temperatura do solo, teores de $\mathrm{C}$ e $\mathrm{N}$ orgânico total, $\mathrm{pH}$, condutividade elétrica, teores de $\mathrm{N}$ mineral $\left(\mathrm{NH}_{4}{ }^{+}\right.$e $\left.\mathrm{NO}_{3}{ }^{-}\right), \mathrm{P}, \mathrm{K}, \mathrm{C}$ e $\mathrm{N}$ da biomassa microbiana, $\mathrm{N}$ potencialmente mineralizável, respiração do solo, $\mathrm{C}$ na biomassa em relação ao $\mathrm{C}$ orgânico total e respiração microbiana em relação à biomassa (VEZZANI et al., 2009).

Nas palavras de Salton et al. (2014), a estrutura adequada do solo é a que permite bom fluxo de água, aeração do seu interior, resistência à erosão e ao tráfico de maquinários, desenvolvimento de 
organismos vivos, e o apropriado desenvolvimento e funcionamento das raízes das plantas. Takasu (2019) considera que a densidade do solo é uma característica muito importante pois permite avaliar indiretamente os atributos como porosidade, condutividade hidráulica, difusividade do ar, além da utilização como indicador do estado da compactação do solo.

Dentre os fatores que podem promover a saúde do solo estão: a utilização de adubos verdes, consorciação, cobertura vegetal, compostagem técnica, população de organismos vivos como as minhocas, adoção de práticas de plantio direto e a rotação de culturas (DUBEY et al., 2020).

\section{Técnicas relacionadas à saúde do solo}

\section{Plantio direto}

A técnica de manejo de solo conhecida como Sistema de Plantio Direto (SPD) se baseia no plantio das culturas sem revolvimento do solo, mantendo a palhada (DEBIASE et al., 2013). Esse sistema de manejo de solo surgiu na década de 1970 devido à falta de controle das erosões hídricas, e da compactação do solo, que eram observados nas propriedades agrícolas. O SPD envolveu esforços de agricultores, pesquisadores e de fabricantes de semeadoras, e buscou inovar os sistemas de manejo do solo por meio da redução da exposição do solo aos efeitos das chuvas, visto que as técnicas de cultivo em nível e terraços eram insuficientes para evitar esses danos nas lavouras (ANDRADE et al., 2010; CASÃO JUNIOR et al., 2012).

Segundo Casão Junior et al. (2012), o pioneiro na implantação do SPD em grande escala foi o produtor Herbert Bartz, de Rolândia - PR. Seu interesse foi motivado em 1971, após uma chuva de 90 mm ocasionar severas erosões, rompimento de terraços e arraste das plântulas pela água em sua lavoura de soja. Com o ocorrido, o produtor buscou alternativas ao sistema de preparo convencional. A implantação e o sucesso do SPD no Paraná geraram reflexos positivos na produção de grãos e na conservação dos solos e água, e foi ampliado para outras regiões do país nos anos seguintes (ANJOS et al., 2010).

O SPD permite alterações significativas no solo, pois diminui seu revolvimento mecânico, sendo este revolvimento restrito somente às linhas de semeadura, e mantém os resíduos das culturas sobre o solo (ANDRADE et al., 2010). Carvalho et al. (2009) afirmam que o maior aporte de resíduos de culturas e a falta de perturbação do solo sob SPD são as forças motrizes que proporcionam a acumulação de carbono no solo. Além disso, a matéria orgânica é considerada um dos principais indicadores da qualidade do solo, pois está relacionada diretamente com os aspectos químicos, físicos e biológicos do solo (MATOSO et al., 2012). Andrade et al. (2010) ainda destacam que com o acúmulo de material orgânico na superfície do solo, a decomposição ocorre mais lentamente, pois diminui a exposição aos microrganismos do solo.

\section{Consórcio de culturas}

O cultivo de plantas em consórcio é uma tecnologia praticada há séculos. É caracterizada pelo cultivo simultâneo, na mesma área agrícola de duas ou mais espécies com características divergentes, no que se refere a arquitetura vegetal, hábitos de crescimento e fisiologia. As espécies selecionadas para o cultivo em consórcio podem ser semeadas ao mesmo tempo, ou terem épocas de implementação 
levemente defasada, compartilhando dos mesmos recursos ambientais durante seus ciclos de vida (PIEDRA-BONILLA et al., 2020). Isto leva a forte interatividade entre as espécies consorciadas e entre elas e o meio ambiente.

A técnica de consorciação tem por objetivo a produção de grãos, forragem para a alimentação animal, cobertura vegetal para a próxima cultura, a reforma de pastagens, e diversos benefícios para a sustentabilidade e consolidação da produção no SPD, principalmente em áreas de Cerrado (TAKASU, 2019), além disso, auxilia no controle de plantas daninhas, e atua maximizando o aproveitamento da água disponível no solo ou do período chuvoso. O tipo de consorcio mais empregado é o que envolve uma gramínea e uma leguminosa, pois as leguminosas liberam nitrogênio no solo advindo do processo de fixação biológica e este nutriente pode ser utilizado pela outra cultura (CHAPAGAIN et al., 2018).

Neste contexto, destacam-se os consórcios que envolvem o milho (Zea mays), pois esta gramínea apresenta características favoráveis para o cultivo consorciado: alto porte das plantas, e altura de inserção das espigas, que, durante a colheita não ocorre a interferência das plantas forrageiras (ALVARENGA et al., 2006). Por apresentar metabolismo fotossintético C4 (SAGE et al., 2011), e rápido crescimento inicial, a cultura do milho é tida como muito competitiva em consórcios (TAKASU, 2019).

Entre as espécies utilizadas na consorciação com o milho, destacam-se as pertencentes à família Poaceae, gramíneas, por possuírem sistema radicular vigoroso e profundo que promove a estruturação do solo, capacidade de absorver nutrientes em camadas profundas do solo, elevada tolerância ao déficit hídrico, além do desenvolvimento em condições ambientais desfavoráveis (SALTON et al., 2014; TAKASU, 2019). As leguminosas (família Fabaceae) também são usadas na consorciação com o milho, como por exemplo, as espécies de feijão (Phaseolus vulgaris), guandu-anão (Cajanus cajan), mucuna-preta (Mucuna aterrima) e crotalária (Crotalaria juncea, C. ochroleuca, e C. spectabilis). Estas, podem aumentar a disponibilidade de nitrogênio no solo para o consórcio, resultando em aumento da produtividade de milho, ou para a cultura em sucessão (OLIVEIRA, 2010; OLIVEIRA et al. 2017).

\section{Cobertura do solo}

De acordo com Calegari et al. (2009), "registros históricos mostram que a prática milenar do uso de plantas de cobertura foi desenvolvida com sucesso por antigas civilizações, mas foi relegada na agricultura moderna, iniciada no século passado". A diversidade nos sistemas de cultivo, principalmente o que se refere a colheita de grãos, incluindo cereais e plantas de cobertura ou culturas de inverno, é importante para reproduzir a estrutura dos ecossistemas naturais, podendo contribuir na melhoria dos serviços dos ecossistemas do solo (FAO et al., 2020).

A diversificação de plantas de cobertura do solo, resultam em benefícios como maior eficiência de uso de $\mathrm{N}$ das culturas (GAUDIN et al., 2015), maior tolerância às condições de seca (GAUDIN et al., 2015), melhor produtividade e estabilidade da produção (BOWLES et al., 2020; GAUDIN et al., 2015), aumento da produtividade por unidade de área (HAUGGAARD-NIELSEN et al., 2012), menor exigência de fertilizantes e pesticidas (SMITH et al., 2008), maior retorno líquido (MEYER-AURICH et al., 2006) e melhor matéria 
orgânica do solo e atividade microbiana (TIEMANN et al., 2015). Além disso, regula o uso da água, reduz a pressão de pragas e doenças, e mantém um melhor rendimento (SCHERR et al., 2008; MALÉZIEUX et al., 2009).

A cobertura vegetal apresenta alta relevância nas regiões dos trópicos, pois o solo é mantido permanentemente coberto por resíduos ou pelas plantas em fase vegetativa. Também apresenta vários atributos físicos, reduzindo as amplitudes térmicas do solo e, colaborando com os processos de germinação de sementes, crescimento radicular, e na absorção de íons (CALEGARI et al., 2009).

\section{Rotação de cultura}

A monocultura promove ao longo do tempo alterações negativas para o sistema produtivo como, por exemplo: a degradação química, física e/ou biológica do solo, aumento de espécies de microrganismos específicos, ocorrência de pestes e doenças, e o aparecimento de espécies daninhas. Estes componentes interferem a relação solo-água-planta, e provoca a redução da fertilidade do solo, e consequentemente menor rendimento das culturas (GONÇALVES et al., 2007). Neste contexto, a rotação de cultura consiste em alternar no tempo, o cultivo de espécies vegetais, preferencialmente com culturas que possuem sistemas radiculares diferentes, e cada espécie deixa um efeito residual positivo para o solo e para a cultura sucessora (GONÇALVES et al., 2007; JAT et al., 2018).

A rotação de diferentes tipos de culturas, com seus diversos tipos de raízes, combinada com a prática de plantio direto, proporciona melhor desenvolvimento nos macroporos do solo por meio dos canais radiculares, colaborando com a infiltração de água em profundidade no perfil do solo. Além disso, aumenta a diversidade microbiana, reduzindo pragas e doenças ocasionadas por patógenos (FAO et al., 2020). Dentre as plantas utilizadas na rotação de culturas, as leguminosas têm papel fundamental, pois podem ser usadas tanto como parte das rotações das culturas quanto consorciadas com outras culturas (RODRIGUES et al., 2020). Os benefícios das leguminosas incluem a fixação biológica do nitrogênio atmosférico, através de uma relação simbiótica com bactérias do solo (JENSEN et al., 2012), redução da emissão de gases de efeito estufa (JENSEN et al., 2012; JEUFFROY et al., 2013), melhora na qualidade do solo, conservação da umidade e supressão de ervas daninhas (KANATAS, 2020).

\section{Terraceamento agrícola}

O terraceamento agrícola é uma das práticas mecânicas de controle da erosão mais difundida entre os agricultores brasileiros. É uma técnica que objetiva a distribuição de terraços nas propriedades agrícolas, levando em consideração as características pluviométricas da região, tais como quantidade, duração e intensidade, e as características do relevo, tais como a rugosidade do terreno, permeabilidade, e profundidade do solo. As práticas de manejo agrícola, como plantio direto, plantio convencional e cultivo mínimo também interferem na escolha do terraço (MACHADO et al., 2017). Esta prática também contribui para a contenção e atraso das águas que carreiam agroquímicos aos mananciais hídricos. Em propriedades de SPD, insumos como fertilizantes, corretivos e herbicidas ficam mais tempo sobre a superfície do solo, 
por isso são mais susceptíveis de serem carreados pela enxurrada, aumentando as poluições de lagoas e rios (PRETI et al., 2017).

\section{CONCLUSÕES}

O emprego da agricultura conservacionista apresenta-se como uma forte opção para o alcance da sustentabilidade agrícola, com a adoção de práticas que podem contribuir com a saúde do solo. Dentre estas destacam-se o plantio direto, o uso de cobertura vegetal, a rotação e/ ou consórcio de culturas e a implantação de terraços. A divulgação destas técnicas pode auxiliar pesquisadores, técnicos e produtores rurais na tomada de decisão para uma produção vegetal eficiente e ambientalmente adequada.

\section{REFERÊNCIAS}

ABHILASH, P. C.; TRIPATHI, V.; EDRISI, S. A.; DUBEY, R. K.; BAKSHI, M.; DUBEY, P. K.; SINGH, H. B.; EBBS, S. D.. Sustainability of crop production from polluted lands. Energy, Ecology and Environment, v.1, n.1, p.54-65, 2016. DOI: https://doi.org/10.1007/s40974-016-0007-x

AHLAWAT, U.; ANU; SINGH, V. K.; SHARMA, V.; SHEKHAWAT, N.; RAI, R. K.. Soil organic matter and its importance for soil health. Food and Scientifis Reports, v.1, n.10, 2020.

ALVARENGA, R. C.; COBUCCI, T.; KLUTHCOUSKI, J.; WRUCK, F. J.; CRUZ, J. C.; GONTIJO NETO, M. M.. A cultura do milho na integração lavoura-pecuária. Sete Lagoas: Embrapa, 2006.

ANDRADE, A. G.; FREITAS, P. L.; LANDERS, J.. Aspectos gerais sobre o manejo e conservação do solo e da água e as mudanças ambientais. In: PRADO, R. B.; TURETTA, A. P. D.; ANDRADE, A. G.. Manejo e conservação do solo e da água no contexto das mudanças ambientais. Rio de Janeiro: Embrapa, 2010.

ANGELAKIS, A. N.; ZACCARIA, D.; KRASILNIKOFF, J.; SALGOT, M.; BAZZA, M.; ROCCARO, P.; JIMENEZ, B.; KUMAR, A.; YINGHUA, W.; BABA, A.; HARRISON, J.A.; GARDUNOJIMENEZ, A.; FERERES, E.. Irrigation of World Agricultural Lands: evolution through the Millennia. Water, v.12, n.5, p.1285, 2020. DOI: https://doi.org/10.3390/w12051285

ANJOS, L. H. C.; PEREIRA, M. G.. Aspectos gerais relacionados à expansão da agricultura brasileira. In: PRADO, R. B.; TURETTA, A. P. D.; ANDRADE, A. G.. Manejo e conservação do solo e da água no contexto das mudanças ambientais. Rio de Janeiro: Embrapa, 2010.

BARBIERI, J. C.; CAJAZEIRA, J. E. R.. Responsabilidade Social Empresarial e Empresa Sustentável: teoria à prática. 3 ed. São Paulo: Saraiva, 2016.

BHATT, R.; KHERA, K. L.. Effect of tillage and mode of straw mulch application on soil erosion in the submontaneous tract of Punjab, India. Soil and Tillage Research, v.88, n.12, p.107-115, 2006. DOI:

https://doi.org/10.1016/i.still.2005.05.004

BIDONE, E. D.; MORALES, P. R. D.. Desenvolvimento Sustentável e Engenharia: enfoque Operacional. Rio de Janeiro: Fundação Ricardo Franco, 2004.
BOWLES, T. M.; MOOSHAMMER, M.; SOCOLAR, Y.; CALDERÓN, F.; CAVIGELLI, M. A.; CULMAN, S. W.; DEEN, W.; DRURY, C. F.; GARCIA, A. G.; GAUDIN, A. C. M.; HARKCOM, W. S.; LEHMAN, M. R.; OSBORNE, S. N.; ROBERTSON, G. P.; SALERNO, J.; SCHMER, M. R.; STROCK, J.; GRANDY, A. S.. Long-Term evidence shows that crop-rotation Diversification increases agricultural resilience to Adverse Growing conditions in North America. One Earth, v.2, n.3, p.284-293, 2020. DOI: https://doi.org/10.1016/j.oneear.2020.02.007

BÜNEMANN, E. K.; BONGIORNO, G.; BAI , Z.; CREAMER, R. R.; DEYN, G.; GOEDE, R.; FLESKENS, L.; GEISSEN, V.; KUYPER, T. W.; MÄDER, P.; PULLEMAN, M.; SUKKEL, W.; GROENIGEN, J. W. V.; BRUSSAARD, L.. Soil quality: a critical review. Soil Biology and Biochemistry, v.120, p.105-125, 2018. DOI: https://doi.org/10.1016/j.soilbio.2018.01.030

BUSARI, M. A.. Conservation tillage impacts on soil, crop and the environment.. International Soil and Water Conservation Research, v.3, n.2, p.119-129, 2015.

CALEGARI, A.; COSTA, A.. Sistemas conservacionistas de uso do solo. In: PRADO, R. B.; TURETTA, A. P. D.; ANDRADE, A. G.. Manejo e conservação do solo e da água no contexto das mudanças ambientais. Rio de Janeiro: Embrapa Solos, 2009. p.279-308

CARVALHO, J. L. N.; CERRI, C. E. P.; FEIGL, B. J.; PICCOLO, M. C.; GODINHO, V. P.; HERPIN, U.; CERRI, C. C.. Conversion of cerrado into agricultural land in the south-western amazon: carbono stocks and soil fertility. Scientia Agricola, v.66, n.2, p.233-241, 2009. DOI: https://doi.org/10.1590/S0103$\underline{90162009000200013}$

CASÃO JUNIOR, R.; ARAÚJO, A. G.; LLANILLO, R. F.. Plantio direto no Sul do Brasil: fatores que fatores que facilitaram a evolução do sistema e o desenvolvimento da mecanização conservacionista. Londrina: IAPAR, 2012.

CHAPAGAIN, T.; PUDASAINI, R.; GHIMIRE, B.; GURUNG, K.; CHOI, K.; RAI, L.; MAGAR, S.; BK, B.; RAIZADA, M. N.. Intercropping of maize, millet, mustard, wheat and ginger increased land productivity and potential economic returns for smallholder terrace farmers in Nepal. Field Crops Research, v.227, n.1, p.91-101, 2018. DOI: https://doi.org/10.1016/j.fcr.2018.07.016

CHAREL, J. M.; VEJAPARA, V. P.; PARMAR, V. S.; BARIA, N.. Perception of farmers about the soil health card. 
International Journal of Current Microbiology and Applied

Sciences, v.7, n.2, p.3233-3236, 2018. DOI:

https://doi.org/10.20546/ijcmas.2018.702.389

CZYZEWSKI, B.; DOBROWOLSKI, D.; MATUSZCZAK, A.; ŚMIDO DA, P.; SZWON, T.. Social sustainability in agricultural farms with differing economic size in the EU countries.

Proceedings of the $\mathbf{2 0 1 8}$ International Scientific Conference "Economic sciences for agribusiness and rural economy", n.1, p.75-80, 2018. DOI:

https://doi.org/10.22630/ESARE.2018.1.9

DEBIASE, H.; FRANCHINI, J. C.; CONTE, O.; BALBINOT JUNIOR, A. A.; TORRES, E.; SARAIVA, O. F.; OLIVEIRA, M. C. N.. Sistemas de preparo do solo: trinta anos de pesquisas na Embrapa Soja. Londrina: Embrapa, 2013.

DEY, A.; MUKHERJEE, S.. Conservation agriculture: future of agriculture. Soil Science, Agrobios Newsletter, v.18, n.4, 2019.

DORAN, J. W.; PARKIN, T. B.. Defining and assessing soil quality. In: DORAN, J. W.; COLEMAN, D. C.; BEZDICEK, D. F.; STEWART, B. A.. Defining soil quality for a sustainable environment. MADISON, W. I.. Soil Science Society of America and American Society of Agronomy, 1-21, 1994. DOI: http://dx.doi.org/10.2136/sssaspecpub35.c1

DUBEY, P. K.; SINGH, G. S.; ABHILASH, P. C.. Adaptive Agricultural Practices: building Resilience in a Changing Climate. Springer Briefs in Environmental Science, 2020.

DURAN, D. C.; ARTENE, A.; GOGAN, L. M.; DURAN, V.. The objectives of sustainable development: ways to achieve welfare. Procedia Economics and Finance, v.26, n.1, p.812817, 2015. DOI: https://doi.org/10.1016/S22125671(15)00852-7

FAO. State of knowledge of soil biodiversity: status, challenges and potentialities, Report 2020. FAO, 2020. DOI: https://doi.org/10.4060/cb1928en

GAUDIN, A. C. M.; JANOVICEK, K.; DEEN, B.; HOOKER, D. C.. Wheat improves nitrogen use efficiency of maize and soybean-based cropping systems.. Agriculture, Ecosystems \& Environment, v.210, n.1, p.1-10, 2015. DOI: https://doi.org/10.1016/j.agee.2015.04.034

GAUDIN, A. C. M.; TOLHURST, T. N.; KER, A. P.; JANOVICEK, K.; TORTORA, C.; MARTIN, R. C.; DEEN, W.. Increasing crop diversity mitigates weather variations and improves yield stability. PloS one, v.10, n.2, 2015. DOI: https://doi.org/10.1371/journal.pone.0113261

GHOLOUBI, A.; EMAMI, H.; ALIZADEH, A.; AZADI, R.. Long term effects of deforestation on soil attributes: case study, Northern Iran. Caspian Journal of Environmental Sciences, v.17, n.1, p.73-81, 2019. DOI:

https://doi.org/10.22124/CJES.2019.3346

GONÇALVES, S. L.; GAUDÊNCIO, C. de A.; FRANCHINI, J. C.; GALERANI, P. R.; GARCIA, A.. Rotação de culturas. Londrina: Embrapa, 2007.

HARIZANOVA-BARTOS, H.; STOYANOVA, Z.. Impact of agriculture on soil pollution in Bulgaria. Economics of agriculture, v.66, n.2, 2019. DOI:

https://doi.org/10.5937/ekoPolj1902375H
HAUGGAARD-NIELSEN, H.; MUNDUS, S.; JENSEN, E. S.. Grassclover undersowing affects nitrogen dynamics in a grain legume-cereal arable cropping system. Field Crops Research, v.136, p.23-31, 2012. DOI:

https://doi.org/10.1016/j.fcr.2012.07.001

HOBBS, P. R.; SAYRE, K.; GUPTA, R.. The role of conservation agriculture in sustainable agriculture. Philosophical Transactions of the Royal Society B: Biological Sciences, v.363, n.1491, p.543-555, 2008. DOI:

https://doi.org/10.1098/rstb.2007.2169

JANKER, J.; MANN, S.; RIST, S.. Social sustainability in agriculture: a system based framework. Journal of Rural Studies, v.65, p.32-42, 2019. DOI:

https://doi.org/10.1016/j.jrurstud.2018.12.010

JENSEN, E. S.; PEOPLES, M. B.; HAUGGAARD-NIELSEN, H. Faba bean in cropping systems. Field crops research, v.115, n.3, p.203-216, 2010. DOI:

https://doi.org/10.1016/j.fcr.2009.10.008

JENSEN, E. S.; PEOPLES, M. B.; BODDEY, R. M.; GRESSHOLF, P. M.; HAUGGAARD-NIELSEN, H.; ALVES, B. J. R.; MORRISON, $M$. J.. Legumes for mitigation of climate change and the provision of feedstock for biofuels and biorefineries: a review. Agronomy for sustainable development, v.32, n.1, p.329-364, 2012. DOI: https://doi.org/10.1007/s13593-011$\underline{0056-7}$

JEUFFROY, M. H.; BARANGER, E.; CARROUÉE, B.; CHEZELLES, E.; GOSME, M.; HÉNAULT, C.; SCHNEIDER, A.; CELLIER, P.. Nitrous oxide emissions from crop rotations including wheat, oilseed rape and dry peas. Biogeosciences, v.10, n.3, p.1787-1797, 2013. DOI: https://doi.org/10.5194/bg-10$\underline{1787-2013}$

KANATAS, P.. Mini-review: the role of crop rotation, intercropping, sowing dates and increased crop density towards a sustainable crop and weed management in arable crops. Agraarteadus, v.31. n.1, p.22-27, 2020. DOI: https://doi.org/10.15159/jas.20.11

LARSON, W. E.; PIERCE, F. J.. The dynamics of soil quality as a measure of sustainable management. Defining soil quality for a sustainable environment, v.35, p.37-51, 1994. DOI: https://doi.org/10.2136/sssaspecpub35.c3

MALÉZIEUX, E.; CROZAT, Y.; DUPRAZ, C.; LAURANS, M.; MAKOWSKI, D.; OZIER-LAFONTAINE, H.; RAPIDEL, B.; TOURDONNET, S.; VALANTIN-MORISON, M. Mixing plant species in cropping systems: concepts, tools and models. A review. Agronomy for Sustainable Development, v.29, p.4362, 2009. DOI: https://doi.org/10.1051/agro:2007057

JAT, M. L; BIJAY-SINGH; STIRLING, C. M.; JAT, H. S.; TETARWAL, J. P.; JAT, R. K.; SINGH, R.; LOPEZ-RIDAURA, S.; SHIRSATH, P. B.. Chapter Four - Soil Processes and Wheat Cropping Under Emerging Climate Change Scenarios in South Asia. Advances in Agronomy, v.148, p.111-171, 2018. DOI: https://doi.org/10.1016/bs.agron.2017.11.006

MACHADO, A. P.; WADT, S. G, P.. Boas práticas agrícolas: terraceamento. Rio Branco: EMBRAPA Acre, 2017.

MATOSO, S. C. G.; SILVA, A. N.; FIORELLI-PEREIRA, E. C.; COLLETA, Q. P.; MAIA, E.. Frações de carbono e nitrogênio de um Latossolo Vermelho-Amarelo distrófico sob diferentes usos na Amazônia brasileira. Acta Amazônica, v.42, n.2, 
p.231-240, 2012. DOI: https://doi.org/10.1590/S0044$\underline{59672012000200008}$

MEYER-AURICH, A.; WEERSINK, A.; JANOVICEK, K.; DEEN, B.. Cost efficient rotation and tillage options to sequester carbon and mitigate GHG emissions from agriculture in Eastern Canada. Agriculture, ecosystems \& environment, v.117, n.2-3, p.119-127, 2006. DOI: https://doi.org/10.1016/j.agee.2006.03.023.

MESQUITA, E. A.; CRUZ, M. L. B.; PINHEIRO, L. R Ó.. Geoprocessamento aplicado ao mapeamento das formas de uso da terra na área de preservação permanente (APP) da lagoa do Uruaú-Beberibe/CE. Revista Geonorte, v.3, n.5, p.1509-1518, 2016

OLIVEIRA, P.. Consórcio de milho com adubos verdes e manejo da adubação nitrogenada no cultivo de feijão em sucessão no sistema Integração Lavoura-Pecuária no Cerrado. Tese (Doutorado em Fitotecnia) - Universidade Federal de São Paulo, Piracicaba, 2010.

OLIVEIRA, M. G. C.; OLIVEIRA, L. F. C.; KUSDRA, G. R. F; DÍAZ, J. L. C.. Desempenho Produtivo da Cultivar de FeijãoComum BRS Esteio em Unidades Demonstrativas na Região Centro-Sul do Paraná. Santo Antônio de Goiás: Embrapa, 2017.

ONU. Organização das Nações Unidas. População mundial ultrapassará de dez bilhões até 2100. 2017.

ONU. Organização das Nações Unidas. Objetivos do desenvolvimento sustentável. 2015.

PARIKH, S. J.; JAMES, B. R. Soil: the Foundation of Agriculture. Nature Education Knowledge, v.3, n.10, 2012.

PIEDRA-BONILLA, E. B.; BRAGA, C.; BRAGA, M. J.. Diversificação Agropecuária: conceitos E Estatísticas No Brasil. Revista de Economia e Agronegócio, v.18, n.2, p.1-28, 2020. DOI: https://doi.org/10.25070/rea.v18i1.9501

PRETI, F.; GUASTINI, E.; PENNA, D.; DANI, A.; CASSIANI, G.; BOAGA, J.; DEIANA, R.; ROMANO, N.; NASTA, P.; PALLADINO, M.; ERRICO, A.; GIAMBASTIANI, Y.; TRUCCHI, P.; TAROLLI, P.. Conceptualization of water flow pathways in agricultural terraced landscapes. Land Degradation \& Development, v.29, n.3, p.651-662, 2017. DOI:

https://doi.org/10.1002/ldr.2764

RAKSHIT, A.. Impact Assessment of Bio Priming Mediated Nutrient Use Efficiency for Climate Resilient Agriculture. In: SHERAZ M. S.. Climate Change and Agriculture in India: Impact and Adaptation. Springer, 2019.

RODRIGUEZ, C.; CARLSSON, G.; ENGLUND, J.; FLOHR, A.; PELZER, E.; JEUFFROY, M.; MAKOWSKI, D.; JENSEN, E. S.. Grain legume-cereal intercropping enhances the use of soilderived and biologically fixed nitrogen in temperate agroecosystems. A meta-analysis. European Journal of Agronomy, v.118, 2020. DOI: https://doi.org/10.1016/j.eja.2020.126077

SAGE, R. F.; ZHU, X. G.. Exploiting the engine of C4 photosynthesis. Journal of Experimental Botany, v.62, n.9, p.2989-3000, 2011. DOI: https://doi.org/10.1093/jxb/err179
2014.

SANTOS, N. C. D. S.; SILVA, W. A. D.. Desafios e possibilidades da sustentabilidade na agricultura. Revista Geo Sertões, v.4, n.7, p.10-25, 2019.

SARKAR, D.; PAL, S.; SINGH, H. B.; YADAV, R. S.; RAKSHIT, A.. Harnessing bio-priming for integrated resource management under changing climate. In: SINGH, H. B.; SARMA, B. K.; KESWANI, C.. Advances in PGPR Research. CAB International, p.349-363, 2017. DOI: https://doi.org/10.1079/9781786390325.0349

SARKAR, D.; KAR, S. K.; CHATTOPADHYAY, A.; SHIKHA; RAKSHIT, A.; TRIPATHI, V. K.; DUBEY, P. K.; ABHILASH, P. C.. Low input sustainable agriculture: A viable climate-smart option for boosting food production in a warming world. Ecological Indicators, v.115, 106412, 2020. DOI: https://doi.org/10.1016/j.ecolind.2020.106412

SARMINAH, S.; KARYATI, K.; KARMINI, K.; SIMBOLON, J.; TAMBUNAN, N.. Rehabilitation and soil conservation of degraded land using sengon (Falcataria moluccana) and peanut (Arachis hypogaea) agroforestry system.

Biodiversitas, v.19, n.1, p.222-228, 2018.

SCHERR, S. J.; MCNEELY, J. A.. Biodiversity conservation and agricultural sustainability: towards a new paradigm of 'ecoagriculture' landscapes. Philosophical Transactions of The Royal Society B: Biological Sciences, v.363, n.1491, p.477-494, 2008. DOI:

https://doi.org/10.1098/rstb.2007.2165

SMITH, R. G.; GROSS, K. L.; ROBERTSON, G. P.. Effects of crop diversity on agroecosystem function: crop yield response. Ecosystems, v.11, n.3, p.355-366, 2008. DOI: https://doi.org/10.1007/s10021-008-9124-5

SINGH, R., SINGH, G. S.. Traditional agriculture: a climatesmart approach for sustainable food production. Energy, Ecology and Environment, v.2, p.296-316, 2017. DOI: https://doi.org/10.1007/s40974-017-0074-7

SINORE, T.; KISSI, E.; ATICHO, A. .The effects of biological soil conservation practices and community perception toward these practices in the Lemo District of Southern Ethiopia. International Soil and Water Conservation Research, v.6, n.2, p.123-130, 2018. DOI: https://doi.org/10.1016/i.iswcr.2018.01.004

SCHOONOVER, J. E.; CRIM, J. F.. An Introduction to Soil Concepts and the Role of Soils in Watershed anagement. Journal of Contemporary Water Research \& Education, v.154, n.1, p.21-47, 2015. DOI: https://doi.org/10.1111/j.1936-704X.2015.03186.x

TAKASU, A. T.. Manejo do solo e consórcio de gramíneas ou leguminosas no desenvolvimento e produtividade do milho primeira safra e feijão de inverno em sucessão no Sistema plantio direto no cerrado. Tese (Doutorado em Agronomia) Universidade Estadual Paulista, 2019.

COSTA, S. T.; BEZERRA, A.; SILVA, M.; NASCIMENTO, A.; PESSOA, L.. Extensão rural para conservação do solo na agricultura familiar. Extensão em Foco, n.20, p.1-13, 2020. DOI: http://dx.doi.org/10.5380/ef.v0i20.64079

TELLES, T. S.; LOURENÇO, M. A. P.; OLIVEIRA, J. F.; COSTA, G. V.; BARBOSA, G. M. C.. Soil conservation practices in a 
watershed in Southern Brazil. Anais da Academia Brasileira de Ciências, v.93, n.3, 2019. DOI:

https://doi.org/10.1590/0001-3765201920180578

THRALL, P. T.; BEVER, J. D.; BURDON, J. J.. Evolutionary change in agriculture: the past, presente and future. Evolutionary applications, v.3, p.405-408, 2010. DOI: https://doi.org/10.1111/j.1752-4571.2010.00155.x

TIEMANN, L. K.; GRANDY, A. S.; ATKINSON, E. E.; MARINSPIOTTA, E.; MCDANIEL, M. D.. Crop rotational diversity enhances belowground communities and functions in an agroecosystem. Ecology letters, v.18, n.8, p.761-771, 2015. DOI: https://doi.org/10.1111/ele.12453

VAN ES, H. A New Definition of Soil. CSA News, v.62, n.10, p.20, 2017. DOI: https://doi.org/10.2134/csa2017.62.1016
VERMA P.; SINGH D.; PATHANIA I. P.; AGGARWAL,

K. Strategies to Improve Agriculture Sustainability, Soil Fertility and Enhancement of Farmers Income for the Economic Development. In: PANPATTE, D.; JHALA, Y.. Soil Fertility Management for Sustainable Development. Springer: Cingapura, 2019. DOI: https://doi.org/10.1007/978-981-13-5904-0 4

VEZZANI, F. M.; MIELNICZUK, J.. Uma visão sobre qualidade do solo. Revista Brasileira de Ciência do Solo, v.33, n.4, p. 743-755, 2009. DOI: https://doi.org/10.1590/S0100$\underline{06832009000400001}$

YAN, F.; NING, J.; SU, F.. Agricultural Evolution: process, pattern and water resource effect. Applied sciences, v.10, n.15, 5065, 2020. DOI:

https://doi.org/10.3390/app10155065

A CBPC - Companhia Brasileira de Produção Científica (CNPJ: 11.221.422/0001-03) detém os direitos materiais desta publicação. Os direitos referem-se à publicação do trabalho em qualquer parte do mundo, incluindo os direitos às renovações, expansões e disseminações da contribuição, bem como outros direitos subsidiários. Todos os trabalhos publicados eletronicamente poderão posteriormente ser publicados em coletâneas impressas sob coordenação da Sustenere Publishing, da Companhia Brasileira de Produção Científica e seus parceiros autorizados. Os (as) autores (as) preservam os direitos autorais, mas não têm permissão para a publicação da contribuição em outro meio, impresso ou digital, em português ou em tradução. 